\title{
Comparison of 0-D, 1-D and 2-D model capabilities for tidal range energy resource assessments
}

\author{
Athanasios Angeloudis*1, Matthew D. Piggott ${ }^{* 2}$, Stephan C. Kramer*3, Alexandros Avdis*4, Daniel Coles ${ }^{* 5}$ \\ Marios Christou ${ }^{\dagger 6}$ \\ *Earth Science \& Engineering \\ Imperial College London, Royal School of Mines, London, UK \\ ${ }^{1}$ a.angeloudis06@imperial.ac.uk \\ ${ }^{2}$ m.d.piggott@imperial.ac.uk \\ ${ }^{3}$ s.kramer@imperial.ac.uk \\ 4a.avdis@imperial.ac.uk \\ ${ }^{5}$ d.coles@imperial.ac.uk \\ $\dagger$ Civil \& Environmental Engineering \\ Imperial College London, Skempton Building, London, UK \\ ${ }^{6}$ marios.christou@imperial.ac.uk
}

\begin{abstract}
Tidal range energy projects present an attractive means for the predictable and large-scale generation of electricity from the marine environment. In particular, proposals are under consideration in UK waters, with their feasibility currently being under high levels of scrutiny. This is due to a combination of potential environmental and socio-economic impacts that are challenging to quantify in the absence of a standardised methodology. At present, numerical models are being developed to provide robust resource/impact assessments and inform future designs. However, modelling inconsistencies in the representation of tidal power plants, operational algorithms, and turbine technology parameters can be observed in the studies to-date. This has made comparisons between proposed designs difficult to accomplish and rely upon. We present a series of models that progressively and consistently introduce spatial dimensions in resource prediction applications. The capabilities and limitations of each of these models are discussed with regard to the evaluation of energy resource and potential hydrodynamic impacts of tidal power plant proposals. Results highlight that a range of hydrodynamic scales should be considered, employing updated parametric models relating to the turbine technology capabilities. These steps will inform optimisation analyses and the robustness of tidal power plant proposals.
\end{abstract}

Index Terms-Tidal Range Energy, Tidal Lagoon, Tidal Barrage, Marine Energy, Resource Assessment

\section{INTRODUCTION}

Tidal range structures are a type of marine renewable energy generation scheme that extracts the potential energy contained in tidal dynamics in areas characterised by a high tidal range. They essentially constitute dams that either span an entire estuarine basin or are positioned against coastlines where the tidal energy resource is sufficient to site turbines within the dam walls in order to viably generate electricity.

A characteristic example of a major existing tidal range structure is the La Rance barrage near Saint-Malo in France, where power has been harnessed from the tides since 1966 [1] using bulb turbines, generating approximately $0.50 \mathrm{TWh} / \mathrm{yr}$. The capacity of the La Rance plant has been superseded by the
Lake Sihwa tidal power station, completed in 2011 in South Korea, with an energy output of approximately $0.55 \mathrm{TWh} / \mathrm{yr}$.

The potential environmental impacts caused by tidal range schemes is a primary concern; coastal structures invariably interfere with the regional hydrodynamics and the local ecological, coastal and estuarine processes. As a result, the development of new tidal range schemes has been delayed by uncertainties over the potential environmental impacts. Addressing these concerns has proved to be particularly challenging for larger schemes, e.g. for a Severn Barrage. In particular, proposals from the Severn Tidal Power Group (1989) and Hafren Power (2010) failed to address the environmental impact induced by the operation of the turbines and sluice gates and were dismissed [2]. Subsequently, alternative options that may be more environmentally-friendly have been proposed through the development of the tidal lagoon concept, where a smaller footprint and hence reduced disruption to existing estuarine processes is possible. Tidal lagoons effectively operate on the same principles as tidal barrages. They are distinguished from barrages by the fact that the majority of their impoundment is artificial. This enables their development in locations that are less environmentally sensitive at the expense of a longer embankment. Barrages, on the other hand, are mainly restricted to estuary mouths and spanning the entire coastal basin width; thus they are more invasive in terms of their effect on estuarine processes.

Assessment of tidal power plant resource and potential impacts relies on the development of numerical tools that simulate their operation over time. These can range from simplified theoretical models, through very simple/cheap numerical algorithms, to more sophisticated multi-dimensional hydrodynamics packages that may require High Performance Computing to be applicable in practice [3]-[6].

Recently, in early 2017, a proposal by Tidal Lagoon Power Ltd (TLP) for a lagoon sited in Swansea Bay has been received favourably by the UK government's independent "Hendry" 
review [7] which advised that multiple significant benefits arise from developing lagoon-based tidal range energy generation technology and associated projects. This highlights an urgent and growing interest in the development of appropriate methodologies for the fundamental design and operational optimisation of larger and multiple tidal range structures [2].

The development of such numerical tools requires an understanding of the limitations and capabilities of tidal power plant operations. However, the interpretation of the predictions from these must also take into account assumptions underpinning the development of the numerical tools themselves. One of the key assumptions often made involves the number of spatial dimensions which are explicitly included within the model. In this work we consider a consistent hierarchy of modelling approaches of varying spatial dimensions, and investigate their application to resource assessment for tidal range energy schemes. In particular, this work is motivated by the lack of studies that directly compare 0-D, 1-D and 2-D modelling approaches in a manner that considers the same technical specifications and tidal power plant operation assumptions.

\section{Methodology}

The operation of tidal range structures relies on generating an artificial tidal phase difference either side of an impoundment, and then enabling the seawater to flow through turbines in the impoundment wall. The potential power $(P)$ generated is fundamentally related to the upstream wetted surface area $\left(A_{s}\right)$ and the square of the water level difference $(H)$ created between the upstream and downstream sides of the separating impoundment:

$$
P \propto A_{s} \times H^{2}
$$

The generation of energy according to the above principle, requires at least four components [8]. Embankments form the outline of the tidal range structure and are designed to impound an area proportional to the energy to be harnessed from the scheme. Turbines are typically located in the deeper sections of the impoundment [2], subject to their size and the local sea-bed geomorphology. The turbines, in turn, generate energy as per the control sequence adopted by the power plant. Sluice gates are located in the impoundment so as to allow the transfer of seawater with minimum obstruction. Finally, to minimise marine transport disruption, locks can be incorporated along the structure to allow vessels to access or exit the impoundment area safely.

The theoretical maximum for the energy produced through the emptying and filling of tidal range structures, neglecting any form of losses, has been investigated by [9] and quantified as

$$
E_{\max }=4 \rho g A_{s} \alpha^{2}
$$

where $E_{\max }$ is the energy generated over a tidal cycle, $\rho$ the density of seawater, $g$ the gravitational acceleration and $\alpha$ the amplitude of the tide at the particular location. Equation 2 was derived by assuming that: (a) the tide is sinusoidal and of amplitude $\alpha$, and (b) the surface area of the impounded basin $\left(A_{s}\right)$ remains constant. The maximum energy is extracted by emptying the basin instantaneously from high water to low water as considered by the double-effect cycle operation of [10]. In most locations, including for the case studies considered here, the dominant tidal constituent is the lunar semi-diurnal, denoted as $M_{2}$. The tidal period of $M_{2}$ is $\approx 12.42 \mathrm{~h}$ and can be typically used as an estimate of the time required for $E_{\max }$ to be generated theoretically, given the amplitude $\alpha$.

\section{A. O-D operation modelling}

In a potential site where the tidal conditions are known, the power plant control algorithms are established, and appropriate formulae that represent the performance of constituent hydraulic structures are available (e.g. turbines and sluice gates), it is possible to simulate the performance of a tidal range structure [11], [12]. The plant operation can be modelled using a water level time series representing the transient downstream water elevations at the site location [3], [13], [14]. In the classical study of [9], it was assumed that (i) the flow through the turbines is at a constant flow rate $Q$ and that (ii) power generation starts and stops at the same prescribed minimum head $h_{\text {min }}$. Since then, developments in 0-D modelling for tidal range structures have been presented by [11], [13]-[15] so that:

- spring-neap conditions and all other relevant tidal signals can be included,

- realistic turbine and sluice gate characteristics can be considered to calculate variable transient flow-rates,

- dynamic control algorithms can be imposed, and

- the variable wetted surface area in the presence of intertidal regions can be parametrised.

Examples of 0-D modelling include successful applications to optimise plant operation parameters [11], estimate the energy output of proposed tidal range structures [16], and assess certain hydro-environmental interactions such as the effect of storm surges on the energy output of tidal range turbines [17].

In accordance with these expected capabilities, a 0-D model is considered here based upon a backward-difference finite difference model adhering to the principles of mass conservation. In essence, for a downstream $\left(\eta_{\mathrm{dn}, i}\right)$ and upstream $\left(\eta_{\mathrm{up}, i}\right)$ water level at an instant in time $t$ corresponding to a timestep $i$, the upstream water level at $i+1$ can be calculated via

$$
\frac{\Delta \eta_{\mathrm{up}}}{\Delta t}=\frac{Q\left(H_{i}\right)+Q_{\mathrm{in}, i}}{A_{s}\left(\eta_{\mathrm{up}, i}\right)}
$$

where $A_{s}\left(\eta_{\mathrm{up}, \mathrm{i}}\right)$ is the wetted surface area of the tidal range structure assuming a constant water level surface of $\eta_{\mathrm{up}, \mathrm{i}}$. $Q_{\text {in }, i}$ corresponds to the sum of inflows/outflows through independent sources such as rivers or outfalls. The water head difference $H_{i}=\eta_{\mathrm{up}, i}-\eta_{\mathrm{dn}, i}$ feeds into $Q\left(H_{i}\right)$ - a function for the total discharge contributions from turbines and sluice gates. As an example, the flow through a hydraulic structure is calculated as in [18]: 


$$
Q\left(H_{i}\right)=C_{d} A_{f} \sqrt{2 g H_{i}},
$$

where $C_{d}$ is a discharge coefficient and $A_{f}$ is the crosssectional flow area in $\mathrm{m}^{2}$. In turn, the power $P$, in $\mathrm{MW}$, produced from a tidal range turbine for a given $H_{i}$ can be expressed as:

$$
P\left(H_{i}\right)=\rho g Q_{T} H_{i} \eta_{o},
$$

where $\rho$ is the fluid density, $Q_{T}$ is the turbine flow rate and $\eta_{o}$ is the overall efficiency factor associated with the turbines in place. In practice, the hydraulic structure flow rates and power output should be represented by hill charts specific to the individual characteristics of the sluice gates and turbines under consideration, thus accounting for their technical constraints [14]. Due to the commercially sensitive nature of turbine performances, it is difficult to acquire detailed hill charts according to the latest turbine technology. The parametrisation of turbines has therefore been accomplished here following the guidelines of [3], who discuss the representation of a doubleregulated bulb turbine designed by Andritz Hydro.

The method followed to represent tidal range turbines is summarised in Table I as a nine-step procedure. The efficiency factors included in step 8 correspond to various components of the turbine itself (e.g. gearbox / drivetrain efficiency $\approx 97.25 \%$, transformer $\approx 99.5 \%$, generator $\approx 97 \%$ etc.), physical aspects (e.g. water friction $\approx 95 \%$ ) as well as design constraints such as the orientation of the turbines relative to the flow direction, in which case we incorporate an additional $10 \%$ reduction for reverse generation.

TABLE I

PARAMETRISATION OF A DOUBLE-REGULATED BULB TURBINE APPLIED IN

\begin{tabular}{|c|c|c|}
\hline$\#$ & Formulations & Description \\
\hline 1 & $S_{p}=\frac{2 \cdot 60 \cdot f_{g}}{G_{p}}$ & $\begin{array}{l}\text { Turbine speed } S_{p}(\mathrm{rpm}) \text {, } \\
\text { where } f_{g} \text { is the grid fre- } \\
\text { quency }(\mathrm{Hz}) \text { and } G_{p} \text { the } \\
\text { generator pole number. }\end{array}$ \\
\hline 2 & $n_{11}=\frac{S_{p} \cdot D}{\sqrt{H}}$ & $\begin{array}{l}\text { Unit speed } n_{11}(\mathrm{rpm}) \text { where } \\
D \text { the diameter }(\mathrm{m}) .\end{array}$ \\
\hline 3 & $Q_{11}= \begin{cases}0.017 n_{11}+0.49, & n_{11} \leq 255 \\
4.75, & n_{11}>255\end{cases}$ & $\begin{array}{l}\text { Unit discharge } Q_{11} \text { from } \\
\text { empirical equations of [14]. }\end{array}$ \\
\hline 4 & $Q^{*}=Q_{11} D^{2} \sqrt{H}$ & $\begin{array}{l}\text { Discharge estimate } Q^{*} \\
\left(\mathrm{~m}^{3} / \mathrm{s}\right) \text { through turbine for } \\
H(\mathrm{~m}) .\end{array}$ \\
\hline 5 & $P^{*}=\min \left(\rho g Q^{*} H, C_{p}\right)$ & $\begin{array}{l}\text { Potential power for } Q^{*} \text { sub- } \\
\text { ject to the turbine capacity } \\
C_{p} \text { (MW). }\end{array}$ \\
\hline 6 & $Q=\frac{P^{*}}{\rho g H}$ & $\begin{array}{l}\text { Correction of } \mathrm{Q} \text { in case of } \\
\text { maximum capacity } C_{p} .\end{array}$ \\
\hline 7 & $\eta_{h}=-0.0019 n_{11}+1.2461$ & $\begin{array}{l}\text { Empirical expression for } \\
\text { hydraulic efficiency [3]. }\end{array}$ \\
\hline 8 & $\eta_{o}=\eta_{1} \cdot \eta_{2} \ldots \eta_{n-1} \cdot \eta_{n}$ & $\begin{array}{l}\text { Consideration of other effi- } \\
\text { ciency factors. }\end{array}$ \\
\hline 9 & $P=\rho g Q H \eta_{o}$ & $\begin{array}{l}\text { Power P (MW) calculated } \\
\text { subject to efficiency losses. }\end{array}$ \\
\hline
\end{tabular}
THE TIDAL RANGE ENERGY RESOURCE ASSESSMENTS

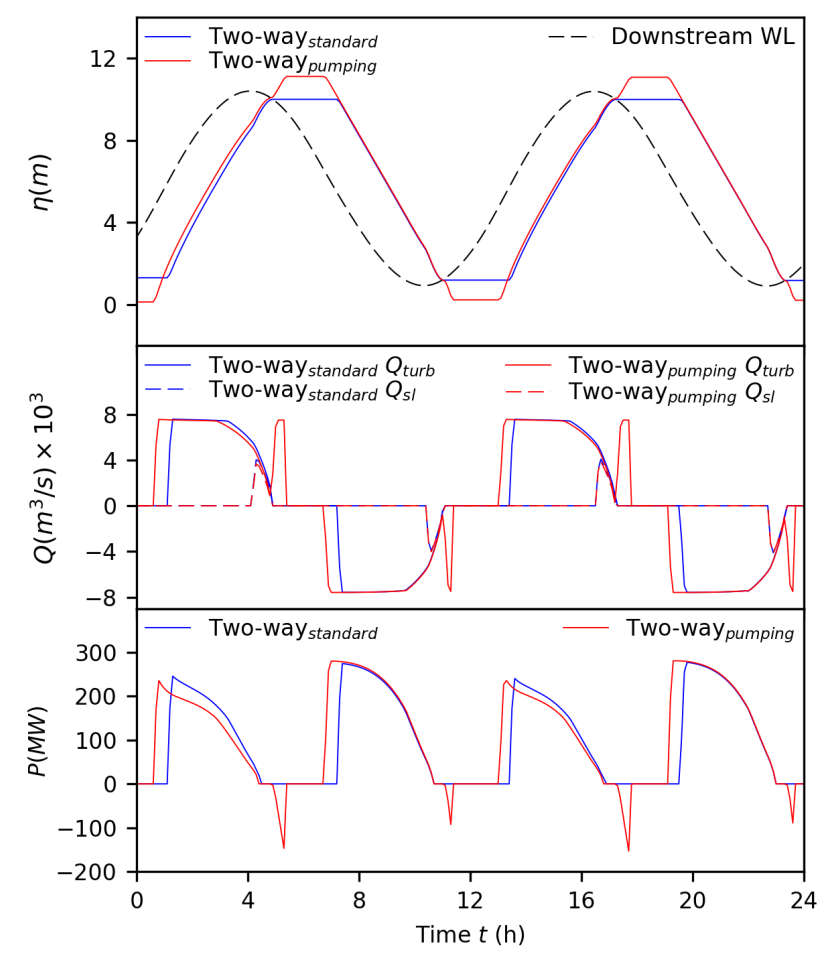

Fig. 1. Example of operation sequence differences between a two-way operation and a two-way operation with pumping, showing (a) water level (with the dashed line showing the downstream water level), (b) discharge and (c) power output.

Ultimately, the flow rate $Q$ and power $P$ are regulated by the plant mode of operation (Figure 1) which will restrict/allow flow through turbines and sluice gates at certain times of the tidal cycle. If the sluice or turbine wicket gates are open, then the discharge is calculated either using Eq. (4) during sluicing or the hill chart parametrisation of Table I while generating. In one-way ebb generation, the incoming tide enters through sluice gates and idling turbines. Once the maximum feasible water level upstream is achieved, the gates are closed until an optimum head $\left(h_{\mathrm{st}}\right)$ develops on the receding tide. Power is then generated until turbines are no longer operating efficiently. For flood generation, the whole process is reversed to generate power during the rising tide. In two-way operation, energy is extracted on both the ebb and flood tide, with sluicing triggered around the times of high and low water.

A schematic representation of the two-way operation algorithms developed for this work is shown in Fig. 1. As shown, Ebb/flood generation can be supplemented with the pumping of water through the turbines to increase the water head difference values further. Therefore, the primary incentive for the use of pumping in tidal range schemes is the noticeable energy gains [16], but also a degree of flexibility in the generation timing. However, the extent of these benefits depends on the pumping efficiency of the bulb turbines in place. For example, an ebb-only operation with pumping has been 
reported in the study of [15] while assessing the energy output of a Swansea Bay lagoon. In Fig. 1 next to the conventional two-way operation algorithm reported previously in [2], we introduce a two-way operation with mitigation pumping, since this is under consideration for ongoing tidal lagoon proposals in the UK. From the standpoint of minimising environmental impact, mitigation pumping has the advantage of preserving the upstream tidal range, thus compensating for intertidal area losses induced by a conventional plant operation.

Even though 0-D modelling has clear computational efficiency advantages, it assumes that the impact of the tidal impoundment itself on the localised tidal levels is negligible. Such an assumption can yield over-optimistic results as reported in [19], depending on the scale and the location of each project. Consequently, it is recommended that analyses should be expanded to couple the regional or even far-field hydrodynamics.

\section{B. 1-D operation modelling: Saint-Venant equations}

We initially consider the solution to the 1-D Saint-Venant equations, to investigate the interaction of tidal range projects with the regional hydrodynamics:

$$
\begin{gathered}
\frac{\partial A}{\partial t}+\frac{\partial(A u)}{\partial x}=0 \\
\frac{\partial u}{\partial t}+u \frac{\partial u}{\partial x}+g \frac{\partial \eta}{\partial x}=-\frac{\tau_{b}}{\rho H_{d}},
\end{gathered}
$$

where $A$ is the flow area and is a function of the water elevation $(\eta)$ according to the cross-sections as in Fig. 2, $u$ the (scalar) depth-averaged horizontal velocity, $H_{d}=\eta+h$ the total water depth where $h$ is the mean water depth, and $\tau_{b}$ represents bed shear stress effects through a Manning $n$ formulation.

The Saint-Venant equations $(6,7)$ are solved here using a finite difference MacCormack method [20], which was implemented for this work in Python. Our implementation has been specifically designed for sensitivity studies of tidal range energy options in idealised geometries. The application of 1-D modelling for tidal energy purposes is not new [21], [22], but has not been extensively applied in the academic literature in relation to tidal energy options while also considering technical constraints due to turbines and sluice gates. In fact, the scale of the proposals (e.g. a Severn Barrage) considered over the past decades required a direct expansion to 2-D modelling as the surrounding hydrodynamics could not be reliably simplified in 1-D. The 1-D operation modelling approach is revisited here to assess its capabilities when applied in projects of varying scale.

Similarly to previous 2-D hydrodynamic studies, e.g. [23], a spatial domain decomposition is adopted to represent tidal range structures. In this case, the upstream and downstream sections are modelled independently as two coupled 1-D models. For barrages, the constituent sections are linked at the respective ends whereas tidal lagoons are treated as junctions to the main channel section. Hydraulic structure flows are relying on the same operation algorithms considered within the 0 -D modelling section. However, the water head differences that drive the turbines and the sluice gates are calculated by the head difference between cells either side of the structure, i.e. from the nodes within a $1 \mathrm{~km}$ distance from the hydraulic structures. In particular, the discharge is uniformly imposed to the incoming/outgoing flows, preserving mass-conservation between coupled sections.

\section{2-D operation modelling: shallow water equations}

The next step towards improving the operation assessment involves extending the hydrodynamic modelling to two and three dimensions, since estuarine and coastal flow conditions can only be accurately reduced to 1-D in limited spatial circumstances. The 2-D coastal modelling was conducted within Thetis (http://thetisproject.org/). Thetis is a (2-D and3D) flow solver for simulating coastal and estuarine flows, implemented using the Firedrake finite element PDE solver framework [24]. In the configuration employed for this work, Thetis solves the non-conservative form of the shallow water equations:

$$
\begin{gathered}
\frac{\partial H}{\partial t}+\nabla \cdot(H \mathbf{u})=0 \\
\frac{\partial \mathbf{u}}{\partial t}+\mathbf{u} \cdot \nabla \mathbf{u}+g \nabla \eta=-\frac{\tau_{b}}{\rho H_{d}},
\end{gathered}
$$

where $\mathbf{u}$ is the depth-integrated velocity vector with components in the horizontal and lateral direction $(u, v)$. Bed shear stress $\left(\tau_{b}\right)$ effects are represented through Manning's formulation as with the 1-D model, in this case extending to two-dimensional velocity components:

$$
\frac{\tau_{b}}{\rho}=g n^{2} \frac{\|\mathbf{u}\| \mathbf{u}}{H_{d}^{\frac{1}{3}}} .
$$

In consideration of the intertidal areas that can influence the tidal power plant performance, wetting and drying effects are represented according to the formulation of [25]. The model is implemented using a discontinuous Galerkin finite element method (DG-FEM), using the $\mathrm{P}_{1 \mathrm{DG}} \mathrm{P}_{1 \mathrm{DG}}$ velocity, pressure finite element pair. A semi-implicit Crank-Nicolson timestepping approach is applied for temporal discretisation with a constant timestep of $25 \mathrm{~s}$. The discretised equations are solved using Newton's nonlinear solver algorithm through the PETSc library [26].

Beyond the imposed water levels at the open boundaries and the river inflows along the coast, the representation of the turbines and sluice gates is implemented following the recent flux-based representation described in [5]. This is combined with the same operation algorithms that were employed for the 0-D and 1-D modelling described in the previous sections. 


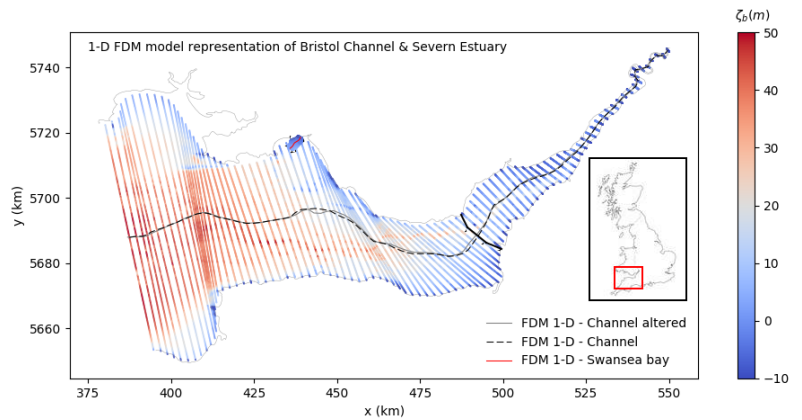

Fig. 2. Representation of the Bristol channel and the Severn estuary in the 1-D models, depicting examples of cross-sections that represent the channel/estuarine bathymetry and the upstream Swansea bay lagoon area. $\zeta_{b}$ denotes the bed elevation in $(\mathrm{m})$.

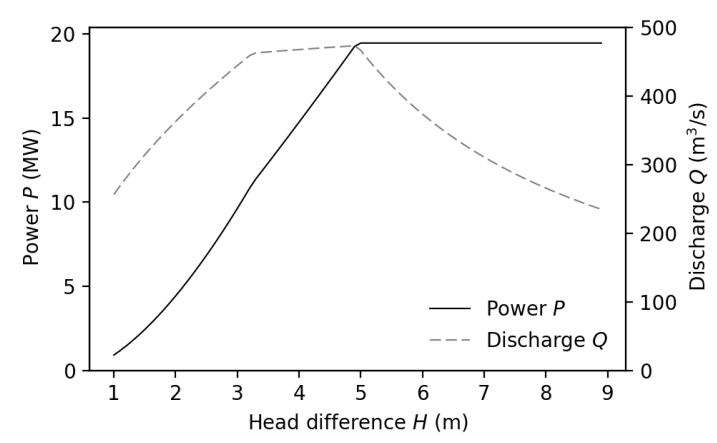

Fig. 3. Bulb turbine hill chart produced according to the sequence presented in Table I for a $7.35 \mathrm{~m}$ diameter turbine with a capacity of $20 \mathrm{MW}$ that is considered for simulations of a tidal lagoon in the Swansea Bay area.

\section{Tidal range structure case studies}

The region of the Bristol Channel and the Severn Estuary in the South West of the UK has been selected for tidal range energy case studies (Fig. 2) on the grounds of the heightened interest to construct tidal range structures in the area. We use two of the most advanced proposals reported to-date as case studies, namely the Swansea Bay tidal lagoon by TLP (Tidal Lagoon Power) and the Severn Barrage STPG (Severn Tidal Power Group) scheme.

The Swansea Bay Lagoon project by TLP was granted planning consent in 2015 and, if constructed, would have an installed capacity of $320 \mathrm{MW}$. It would become the largest tidal range project to-date and, contrary to earlier proposals, it

TABLE II

TIDAL POWER PLANT CASE STUDY SPECIFICATIONS

\begin{tabular}{l|c|c}
\hline Specifications & $\begin{array}{c}\text { Swansea Bay } \\
\text { Lagoon }\end{array}$ & $\begin{array}{c}\text { Severn Barrage } \\
\text { STPG }\end{array}$ \\
\hline Surface area $A_{s}\left(\mathrm{~km}^{2}\right)$ & 11.6 & 573 \\
Capacity $(\mathrm{MW})$ & 320 & 8640 \\
Turbine $D(\mathrm{~m})$ & 7.35 & 9.00 \\
Generator Poles $G_{p}$ & 95 & 142 \\
Turbine No. & 16 & 216 \\
Sluice Area $\left(m^{2}\right)$ & 800 & 35000 \\
\hline
\end{tabular}
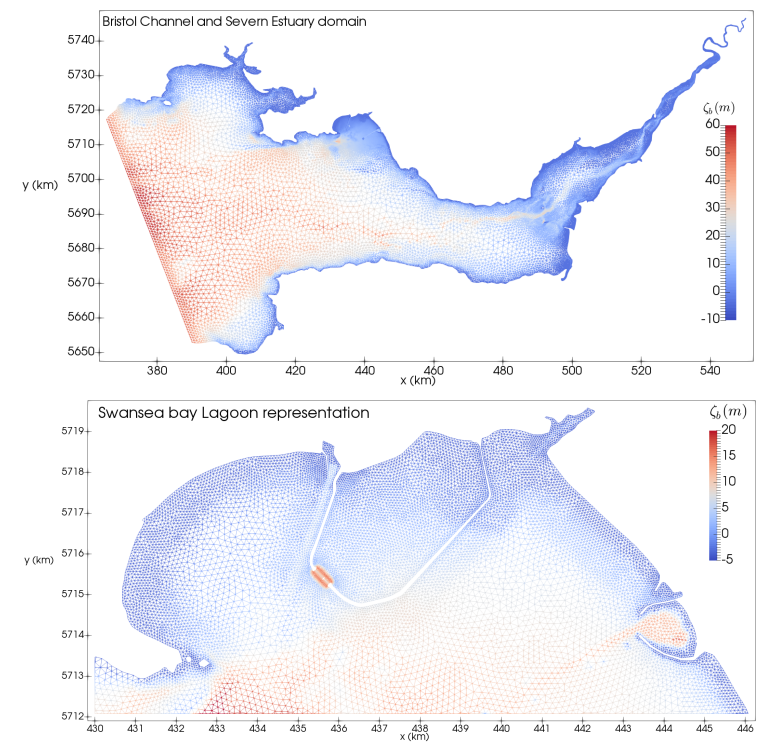

Fig. 4. (a) Example of the computational domain extent and mesh generated to study the operation of tidal range structures for the 2-D simulations using Thetis and (b) mesh detail around the Swansea bay lagoon simulation. $\zeta_{b}$ denotes the bed elevation in $(\mathrm{m})$.

is designed to function through a two-way operation sequence to reduce power generation intermittency. At the time of writing, outstanding details are negotiated with the UK Government and appropriate environmental agencies. Therefore, construction could commence as early as 2018.

A Cardiff-Weston (Severn) barrage was a detailed proposal that was put forward in 1989. Consisting of an $8640 \mathrm{MW}$ capacity, it would dwarf existing tidal power stations and is treated here as an example of a large-scale scheme. The configuration of both designs is schematically illustrated in Fig. 2. Their main specifications considered in our modelling applications are summarised in Table II. These values were in turn fed into the parameters outlined in Table I to produce hill charts as shown in Fig. 3.

The domain representation in 1-D considers bathymetric cross-sections that describe the flow-area at estuary section nodes while taking into account wetting and drying effects in the transverse direction to the flow as well as alterations to the main channel from the introduction of tidal range structures. For the 2-D Thetis simulations, the mesh generation software described in [27], [28] was employed in order to produce the multi-scale unstructured triangular meshes that are refined in areas of interest, such as in the vicinity of the tidal range structures as seen in Fig. 4 for the Swansea Bay lagoon case.

The operational parameters selected remain constant for all the simulations. The main input values that dictate the control algorithms of Fig. 1 are given in Table III for completeness. For these preliminary simulations, pumping periods consider a constant flowrate and an efficiency factor of $70 \%$. 
TABLE III

TIDAL POWER PLANT OPERATIONAL PARAMETERS

\begin{tabular}{l|c}
\hline Parameters & Input values \\
\hline Sluice discharge coefficient $C_{d_{s}}$ & 1.00 \\
Turbine discharge coefficient $C_{d_{t}}$ & 1.36 \\
Starting head difference $h_{\mathrm{st}}(\mathrm{m})$ & 4.50 \\
Minimum operational head $h_{\min }(\mathrm{m})$ & 1.00 \\
Holding mode duration $t_{h}(\mathrm{~h})$ & 3.00 \\
Pumping duration $t_{p}(\mathrm{~h})$ & 0.60 \\
Pumping boost head target $h_{b}(\mathrm{~m})$ & 1.70 \\
Pumping efficiency $\eta_{p}(\mathrm{~m})$ & 0.70 \\
\hline
\end{tabular}

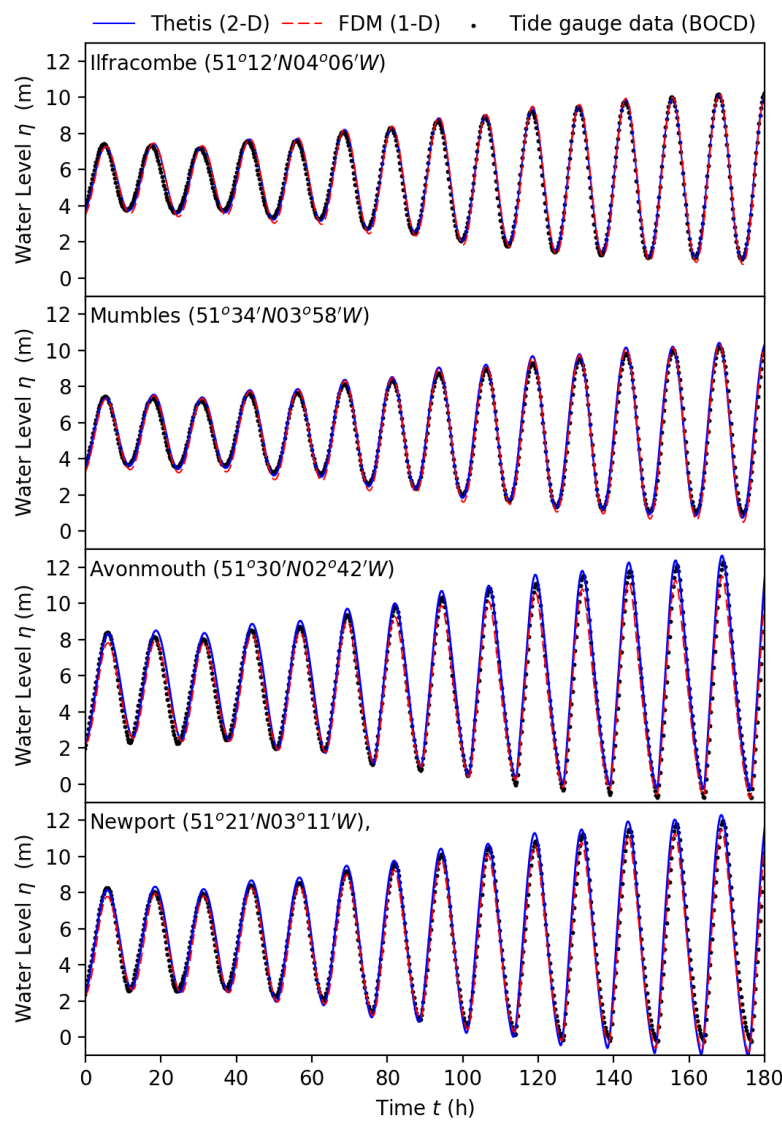

Fig. 5. Agreement between 1-D and 2-D model predictions against tide gauge data for established hydrodynamics in the Severn Estuary and the Bristol Channel.

TABLE IV

Normalised Root MEAn SQUARE DEVIATION (NRMSD) AND THE $R^{2}$ CORRELATION COEFFICIENT AMONG OBSERVED AND PREDICTED WATER LEVELS AT TIDE GAUGE STATIONS ALONG THE BRISTOL CHANNEL FOR THE Thetis 2-D SIMULATIONS

\begin{tabular}{lll}
\hline Location & NRMSD & $R^{2}$ \\
\hline Mumbles & 0.0279 & $99.4 \%$ \\
Ilfracombe & 0.0251 & $99.5 \%$ \\
Hinkley-Point & 0.0423 & $99.0 \%$ \\
Newport & 0.0363 & $99.0 \%$ \\
Avonmouth & 0.0341 & $99.3 \%$ \\
\hline
\end{tabular}

\section{RESULTS \& DISCUSSION}

\section{A. Model application and resource assessment}

Simulations, initially subjected to $48 \mathrm{~h}$ of spin-up time with respect to energy production, then spanned a full lunar month's period between 6/5/2003 and 6/6/2003 to include variable plant performance over spring-neap conditions. The 1-D and 2-D models were tidally forced using eight constituents at the seaward boundaries from the TPXO database [29]. The objective has been to produce comparative results for the three modelling approaches under realistic operating conditions. The starting simulation date was therefore arbitrarily selected and is reported here for completeness. The reliability of the predictions can be affirmed by comparisons with the observed water level time series from the UK's Tide Gauge Network as demonstrated in Fig. 5. In addition, correlation statistics for the 2-D model simulations are summarised in Table IV For this preliminary study, a satisfactory agreement for both 1-D and 2-D models is shown in the Swansea Bay area. Nonetheless, the accuracy of the 1-D model slightly deteriorates at tide gauges within the Severn Estuary. This is expected, given the complexity of the flows in the estuary itself that are simply not properly accounted for in 1-D. A constant value of Manning's $n$ value of 0.025 was imposed. In turn, the 0 -D model was forced using water level time series at the proposed turbine location of the Swansea Bay lagoon and the Severn barrage respectively, drawn from the Thetis 2-D simulation of an unaltered Bristol Channel and the Severn Estuary. Indicatively, for the same simulation time of one month, the 0-D, 1-D and 2D simulations required $0.01 \mathrm{~h}, 0.15 \mathrm{~h}$ and $48.0 \mathrm{~h}$ respectively. The differences in computational time should also reflect the resolution of the models. The 1-D model featured a constant $\Delta x$ of $500 \mathrm{~m}$ whereas the 2-D simulations required a higher resolution with a minimum element length of $20 \mathrm{~m}$ in areas of interest and a constant timestep to consistently represent and control the power plant operation.

The tidal power plant operation over time can be seen in Fig. 6 through the water levels, sluice and turbine flow rates and power predicted by the $0-\mathrm{D}, 1-\mathrm{D}$ and 2-D models. A similar trend is predicted as with previous work by [2]. In the case of the Swansea Bay lagoon, there is an overall qualitative agreement between the three modelling approaches, even though the 2-D approach suggests a greater deficit in the energy output predictions. The 0-D approach overestimates the power output peaks, with 1-D being somewhere in between the other two approaches. More noteworthy differences can be observed for the predictions regarding the Severn Barrage where the upstream water level predictions deviate significantly as these can only be poorly represented in the 0-D and 1-D simplifications. In this case, the 0-D approach leads to extensive overestimations of the energy output, while 1-D appears to be more promising (Fig. 6b) as some effects on the phases and amplitudes of the downstream water levels are captured in the predictions. Given the discrepancies between the upstream predictions of the 0-D, 1-D and 2-D approaches, it is understandable why studies recommend an expansion to 


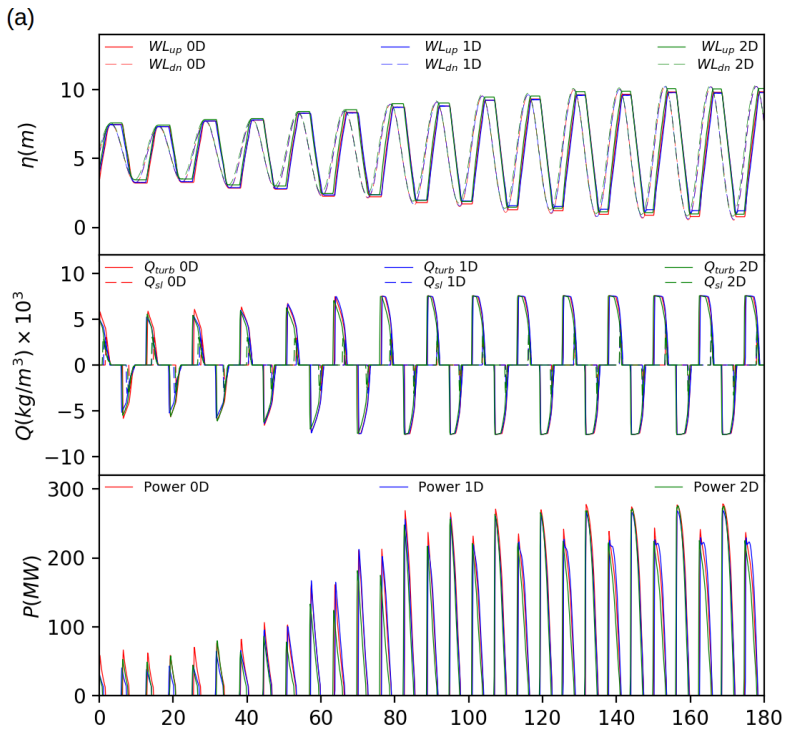

(b)

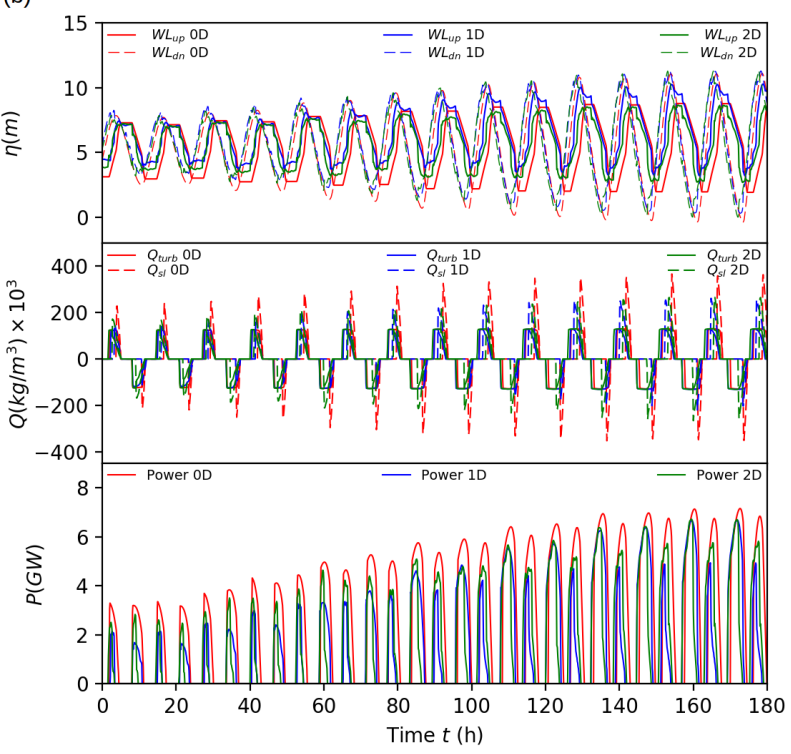

Fig. 6. Comparison between 0-D, 1-D and 2-D model predictions for structure-adjacent average water levels, hydraulic structure flowrates and power output from the operation of (a) the Swansea Bay lagoon and (b) the Severn Barrage under a convetional two-way operation.

far-field coastal modelling studies when dealing with largescale projects [30].

A crucial factor for the feasibility of tidal range structures is their annual energy output as it will dictate their economic viability. There is no concrete methodology for assessments of the annual energy output, even though there have been a number of approaches employed to-date, e.g. [2], [5], [31]. Here we aim to produce normalised results as a fraction of the respective 0-D, 1-D and 2-D model predictions divided by the annual potential energy in an effort to produce a non-dimensional indicator as in [9]. In preceding studies, this involved the calculation of the energy output for a tidal
TABLE V

ANNUAL ENERGY PREDICTION BASED ON A LUNAR MONTH'S SIMULATION SCALED ACCORDING TO THE ANNUAL THEORETICALLY POTENTIAL ENERGY AVAILABLE BETWEEN 05/2003 - 05/2004

\begin{tabular}{l|c|c}
\hline Simulation & $\begin{array}{c}\text { Swansea Lagoon } \\
(T W h / y r)\left(E / E_{\max }\right)\end{array}$ & $\begin{array}{c}\text { Severn Barrage STPG } \\
(\mathrm{TWh} / \mathrm{yr})\left(E / E_{\max }\right)\end{array}$ \\
\hline Theoretical (Eq.(2)) & $0.943(100 \%)$ & $62.22(100 \%)$ \\
\hline 0-D Two-way $_{\text {standard }}$ & $0.445(47 \%)$ & $20.71(33 \%)$ \\
1-D Two-way $_{\text {standard }}$ & $0.436(46 \%)$ & $11.50(18 \%)$ \\
2-D Two-way $_{\text {standard }}$ & $0.411(44 \%)$ & $11.73(19 \%)$ \\
\hline 0-D Two-way & $0.530(56 \%)$ & $22.41(36 \%)$ \\
1-D Two-waypump $_{\text {pumping }}$ & $0.512(54 \%)$ & $11.57(19 \%)$ \\
2-D Two-waypumping & $0.475(50 \%)$ & $11.18(18 \%)$ \\
\hline
\end{tabular}

cycle of mean tidal conditions or simulating full spring-neap cycles and extrapolating the results to the year assuming that the spring-neap simulations are representative, e.g. [2], [32]. There, the average amplitude of the simulated period was used to calculate the annual potential energy. Instead, the tidal range here was calculated and used to estimate the potential energy of each individual cycle through Eq.(2).

If we extrapolate the potential energy contained within the simulated lunar month period to a year, this corresponds to 0.871 and $57.70 \mathrm{TWh} / \mathrm{yr}$ for the Swansea lagoon and the Severn Barrage respectively. However, considering the tidal range for all the cycles in the year, the values obtained amount to 0.943 and $62.22 \mathrm{TWh} / \mathrm{yr}$ (Table V). Therefore a correction factor has been introduced of 1.08 to compensate for the energy predictions drawn from the lunar month in Table V. It should be remarked that the annual energy output is desirable since it can be used to assess the financial viability of different schemes. For the theoretical potential energy, the entire upstream surface area (Table II) has been used in the calculations using Eq. (2), and thus the potential energy does not take into account the transient surface area variability due to the presence of intertidal areas upstream.

The predictions in Table $\mathrm{V}$ are also dependent upon the specifications of the schemes (Table II) as well as the operational characteristics (Table III). This has been outlined previously by [2]. Recent corrections in the turbine hillchart representation, namely in the form of variable hydraulic efficiency included within the sequence of Table I appear to have a significant impact. This corresponds to current annual predictions appearing to be more conservative in 0-D and 2-D than before. However, it should be noted that direct comparisons should be facilitated over the same lunar month's period and for identical operational and turbine characteristics in order to adequately assess the two studies' modelling strategy. Consequently, the provision of technical specifications, model boundary conditions, parameters and assumptions are essential for the reproducibility of the results in subsequent tidal range studies and in order to preserve consistency for benchmarking purposes as the tidal range energy industry develops.

The data presented in Table V suggests that the bidirectional operation of relatively small-sized tidal range structures can 


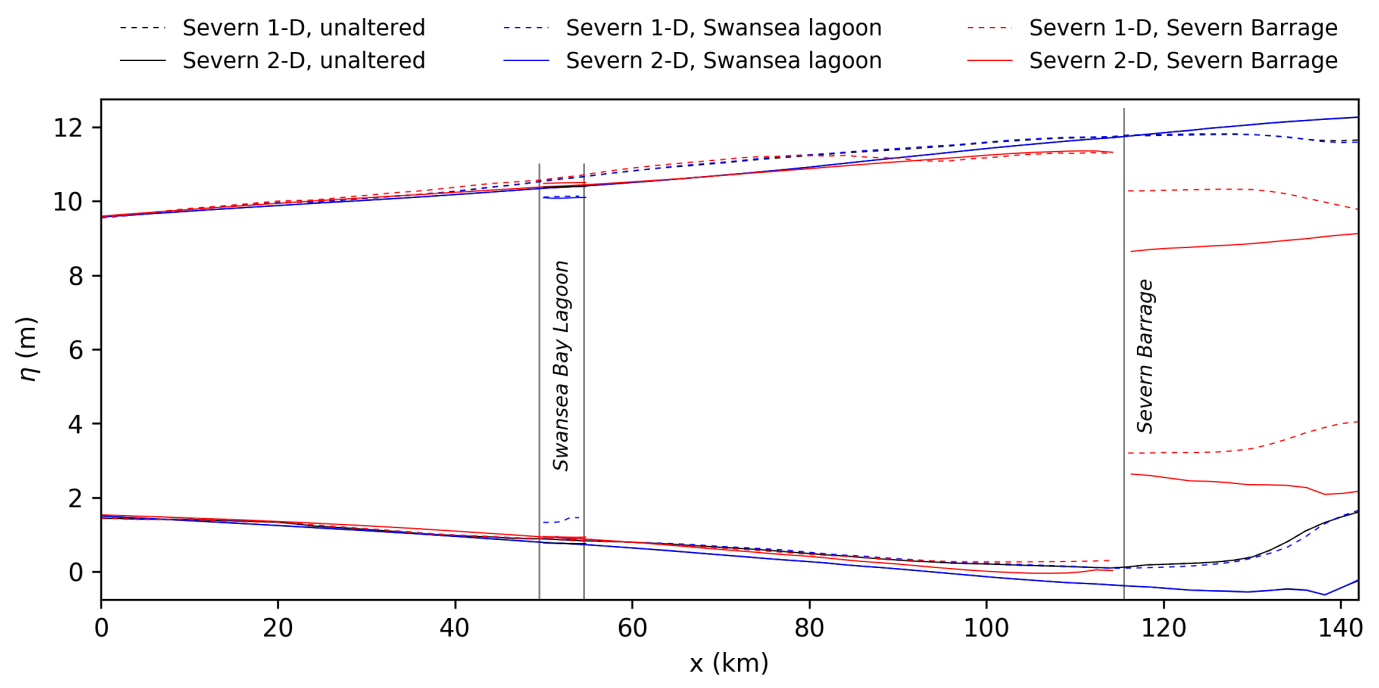

Fig. 7. Impact of tidal range schemes of varying scale as predicted at the centreline of the Bristol Channel and Severn Estuary by 1-D (FDM) and 2-D (Thetis) modelling.

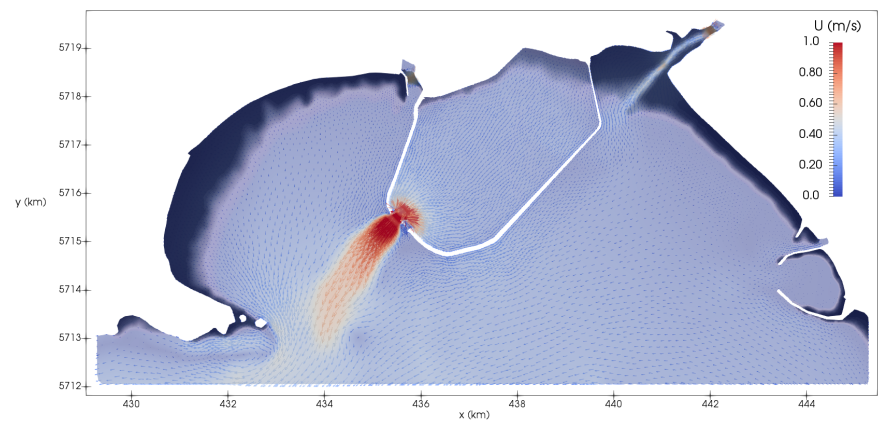

Fig. 8. Prediction of turbine wake using the coastal model Thetis. Hydrodynamic effects such as velocity patterns can only be appreciated in 2-D and 3-D numerical modelling approaches.

correspond to significant energy gains as supported by earlier work of [16]. The pumping benefit for larger scale projects when coupled with the 1-D or 2-D hydrodynamics are contradictory to $0-\mathrm{D}$ results in these preliminary simulations. In larger schemes, a greater amount of energy is required to yield a noticeable head difference and this is further deteriorated by the fact that pumping against lower head differences is markedly inefficient as reported in previous studies [16]. The 1-D and 2-D models predict proportionally greater losses due to hydrodynamic effects (Table V). An appreciation of the pumping benefits should be examined in more detail, as it has been understudied in the literature.

\section{B. Environmental impact assessment}

The environmental impact of tidal range structures has been extensively examined, though unlike energy outputs, effects on water quality and geomorphology can realistically only be fully captured in 2-D, if not fully 3-D modelling methods. In particular, the velocity and suspended sediment

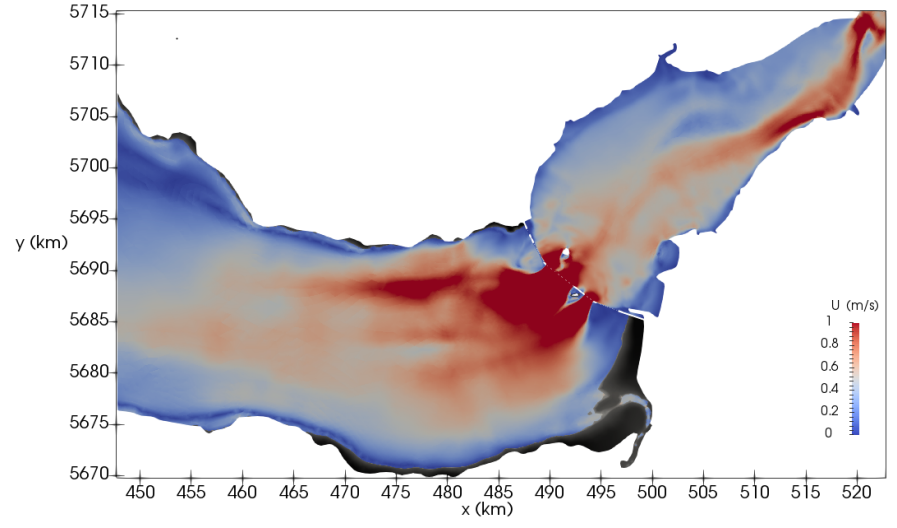

Fig. 9. Prediction of turbine wake using the coastal model Thetis for the Severn Barrage STPG scheme, demonstrating extended wake effects for projects of a larger scale.

concentration measurements at the Lake Sihwa tidal power plant [33] indicate at times an uneven distribution across the water column. Similarly, the 3-D hydrodynamic structure presented in the vicinity of turbine sections by experimental data and CFD studies undertaken at lab-scale conditions [34], [35], also suggest near- to medium- field impacts through jetinduced erosion.

The 0-D model does not provide insights into downstream effects, but could be used to estimate the upstream tidal range, intertidal area losses and mean water level changes upstream as with the indicators introduced in [2], as it considers the basic operation modes of the power plant over time. These estimates will be subject to the underlying 0-D model assumptions, such as constant water levels upstream, an assumption that is clearly invalid at large-scale projects as in Fig. 7 for the Severn Barrage. 
The 1-D model predictions are superior to 0-D in their capability to capture downstream alterations in the tidal resonance, tidal phase as well as cross-sectionally averaged velocity trends. It is also of interest that a water level increase in the medium to far-field range arises as indicated in Fig. 7. This increase has not always been captured in 2-D models when the computational domain has not been sufficient to ensure that the effects of the tidal range structure operation at the seaward boundaries is negligible [12], [23]. In fact, this is applicable to the Thetis computational domain (Fig. 3) used in this study for the Severn Barrage; where the water level increase effects are underestimated in comparison to far-field studies that extend all the way to the continental shelf break where the tide is generated e.g. [4]. While the particular domain is insufficient to rigorously assess the hydrodynamic impacts of a Severn Barrage, the power predicted at the site is independent of the far-field effects downstream and was therefore not extended for simplicity [11] with the interested reader directed to associated studies [30].

The performance of the 1-D model in predicting upstream water level effects is adequate for the Swansea Bay lagoon as it closely follows the 2-D predictions, but is grossly insufficient for the complexity of the flow developed upstream of a larger barrage (Fig. 7).

A rigorous assessment of the impacts would require an extension to at least 2-D as shown by preceding work [8] and demonstrated by the hydrodynamics results of Thetis reported here (Fig. 8-9), which capture some of the depth-averaged wake effects and ensuing vortices. The latter are particularly pronounced for formulations that conserve both the fluid mass and momentum across the hydraulic structures.In addition, an expansion to multi-scale and 3-D modelling will be essential to account for potential stratification and water quality alterations across the water column downstream and upstream. These cannot be accounted for even in 2-D due to modelling limitations.

\section{CONCLUSION}

We have outlined the capabilities of three modelling approaches, ranging from simple theoretical principles all the way to unstructured hydrodynamic coastal modelling in 2-D, so as to conduct tidal range energy resource assessments.

Results suggest that there are a lot of factors that come into play when it comes to the resource assessments, spanning (a) the variability of the tides and available potential power, (b) the impact of the impoundment structure on the established flow conditions, (c) the constraints and the accuracy of turbine performance parametrisations, and (d) the regulation efficiency of the power plant constituent hydraulic structures. As we gradually refine the software and extend to additional dimensions, a distinct feedback on the extracted energy predictions is revealed. However, it is shown that to a certain extent this is captured even by simplified 1-D models which could be used for optimisation purposes on the grounds of their computational efficiency; in conjunction with even more simple 0-D models.
We have also discussed that for rigorous environmental impact assessments, 2-D and 3-D multi-dimensional models are essential considering the complexity of coastal processes such as those related to density stratification and sediment transport. This is because the localised 2-D and 3-D effects by turbine and sluice gates will invariably play a significant role.

The Thetis functionality regarding both the hydrodynamics and turbine operation is consistent with the current stateof-the-art in 2-D modelling in this area. In future work we will exploit the model scalability towards increased resolution in areas of interest. These developments will be undertaken in the context of an iterative design optimisation framework where we explore the mitigation and optimisation of marine structures. In particular, there is scope to improve these environmental impact assessments by extending to 3-D modelling, which at present remains in its infancy with reference to tidal power plant assessment.

\section{ACKNOWLEDGMENT}

The authors would like to acknowledge the financial support of EPSRC under grant EP/M011054/1, and an EPSRC Impact Acceleration award made under project EP/K503733/1.

\section{REFERENCES}

[1] A. Roberts, B. Thomas, P. Sewell, Z. Khan, S. Balmain, and J. Gillman, "Current tidal power technologies and their suitability for applications in coastal and marine areas," Journal of Ocean Engineering and Marine Energy, vol. 2, no. 2, pp. 227-245, 2016.

[2] A. Angeloudis and R. A. Falconer, "Sensitivity of tidal lagoon and barrage hydrodynamic impacts and energy outputs to operational characteristics," Renewable Energy, 2016.

[3] G. Aggidis and O. Feather, "Tidal range turbines and generation on the solway firth," Renewable Energy, vol. 43, pp. 9 -17, 2012.

[4] J Wolf, I. A. Walkington, J Holt, and R Burrows, "Environmental impacts of tidal power schemes," Proceedings of the ICE Maritime Engineering, vol. 162, no. 4, pp. 165-177, 2009.

[5] A. Angeloudis, R. Falconer, S. Bray, and R. Ahmadian, "Representation and operation of tidal energy impoundments in a coastal hydrodynamic model," Renewable Energy, vol. 99, 2016.

[6] S. P. Neill, P. E. Robins, A. Angeloudis, S. Ward, I. Masters, M. J. Lewis, R. A. Falconer, M. Piano, A. Avdis, G. Aggidis, P. Evans, T. A. Adcock, M. D. Piggott, A. Zidonis, and R. Ahmadian, "Tidal range energy resource and optimization - past perspectives and future challenges," Renewable \& Sustainable Energy Reviews, submitted, 2017

[7] C. Hendry, "The role of tidal lagoons," UK Government, Tech. Rep., 2017.

[8] M. Kadiri, R. Ahmadian, B. Bockelmann-Evans, W. Rauen, and R. Falconer, "A review of the potential water quality impacts of tidal renewable energy systems," Renewable and Sustainable Energy Reviews, vol. 16, no. 1, pp. 329-341, 2012.

[9] D. Prandle, "Simple theory for designing tidal power schemes," Advances in water resources, vol. 7, no. 1, pp. 21-27, 1984.

[10] A. Lisboa, T. Vieira, L. Guedes, D. Vieira, and R. Saldanha, "Optimal analytic dispatch for tidal energy generation," Renewable Energy, vol. 108, pp. $371-379,2017$.

[11] A. Angeloudis, R. Ahmadian, R. A. Falconer, and B. BockelmannEvans, "Numerical model simulations for optimisation of tidal lagoon schemes," Applied Energy, vol. 165, pp. 522-536, 2016.

[12] T. A. Adcock, S. Draper, and T. Nishino, "Tidal power generation - a review of hydrodynamic modelling," Proceedings of the Institution of Mechanical Engineers, Part A: Journal of Power and Energy, vol. 0, no. 0, pp. 1-17, 2015.

[13] R. Burrows, I. Walkington, N. Yates, T. Hedges, J. Wolf, and J. Holt, "The tidal range energy potential of the west coast of the united kingdom," Applied Ocean Research, vol. 31, no. 4, pp. 229-238, 2009. 
[14] G. Aggidis and D. Benzon, "Operational optimisation of a tidal barrage across the mersey estuary using 0-d modelling," Ocean Engineering, vol. 66, pp. 69-81, 2013

[15] S. Petley and G. Aggidis, "Swansea bay tidal lagoon annual energy estimation," Ocean Engineering, vol. 111, pp. 348-357, 2016.

[16] N. Yates, I. Walkington, R. Burrows, and J. Wolf, "The energy gains realisable through pumping for tidal range energy schemes,' Renewable Energy, vol. 58, pp. 79-84, 2013.

[17] M. Lewis, A. Angeloudis, P. Robins, P. Evans, and S. Neill, "Influence of storm surge on tidal range energy," Energy, vol. 122, pp. 25 -36, 2017.

[18] A. Baker, "Tidal power," IEE Proceedings A Physical Science, Measurement and Instrumentation, Management and Education, Reviews, vol. 134 , no. 5 , p. 392, 1987.

[19] N. Yates, I. Walkington, R. Burrows, and J. Wolf, "Appraising the extractable tidal energy resource of the uk's western coastal waters," Philosophical Transactions of the Royal Society A: Mathematical, Physical and Engineering Sciences, vol. 371, no. 1985, 2013.

[20] J. C. Tannehill, D. A. Anderson, and R. H. Pletcher, "Computationa fluid dynamics and heat transfer," Series in Computational and Physical Processes in Mechanics and Thermal Sciences, 1997.

[21] H. Suckling, D. Bickley, and S. Ryrie, "Interaction between hydrodynamic effects and control policies in a tidal power scheme," Applied Mathematical Modelling, vol. 16, no. 8, pp. 423-430, 1992.

[22] S. P. Neill, E. J. Litt, S. J. Couch, and A. G. Davies, "The impact of tidal stream turbines on large-scale sediment dynamics," Renewable Energy, vol. 34, no. 12, pp. $2803-2812,2009$.

[23] J. Xia, R. A. Falconer, and B. Lin, "Hydrodynamic impact of a tida barrage in the severn estuary, uk," Renewable Energy, vol. 35, no. 7 , pp. 1455-1468, 2010.

[24] F. Rathgeber, D. A. Ham, L. Mitchell, M. Lange, F. Luporini, A. T. T. Mcrae, G.-T. Bercea, G. R. Markall, and P. H. J. Kelly, "Firedrake: Automating the finite element method by composing abstractions," ACM Trans. Math. Softw., vol. 43, no. 3, 24:1-24:27, Dec. 2016.

[25] T. Kärnä, B. de Brye, O. Gourgue, J. Lambrechts, R. Comblen, V. Legat, and E. Deleersnijder, "A fully implicit wettingdrying method for dg-fem shallow water models, with an application to the scheldt estuary," Computer Methods in Applied Mechanics and Engineering, vol. 200, no. 5, pp. 509-524, 2011

[26] S. Balay, S. Abhyankar, M. F. Adams, J. Brown, P. Brune, K. Buschelman, L. Dalcin, V. Eijkhout, W. D. Gropp, D. Kaushik, M. G. Knepley, L. C. McInnes, K. Rupp, B. F. Smith, S. Zampini, H. Zhang, and H. Zhang, "PETSc users manual," Argonne National Laboratory, Tech. Rep. ANL-95/11 - Revision 3.7, 2016.

[27] A. Avdis, A. S. Candy, J. Hill, S. C. Kramer, and M. D. Piggott, "Efficient unstructured mesh generation for marine renewable energy applications," Renewable Energy, submitted, 2017.

[28] A. Avdis, T. C. Jacobs, L. S. Mouradian, J. Hill, and D. M. Piggott, "Meshing ocean domains for coastal engineering applications," ECCOMAS, 2016

[29] G. D. Egbert and S. Y. Erofeeva, "Efficient inverse modeling of barotropic ocean tides," Journal of Atmospheric and Oceanic Technology, vol. 19, no. 2, pp. 183-204, 2002.

[30] J. Zhou, S. Pan, and R. a. Falconer, "Optimization modelling of the impacts of a severn barrage for a two-way generation scheme using a continental shelf model," Renewable Energy, vol. 72, no. 0, pp. 415427, 2014.

[31] J. Xia, R. a. Falconer, and B. Lin, "Impact of different operating modes for a severn barrage on the tidal power and flood inundation in the severn estuary, uk," Applied Energy, vol. 87, no. 7, pp. 2374-2391, 2010

[32] J. Xia, R. A. Falconer, B. Lin, and G. Tan, "Estimation of annual energy output from a tidal barrage using two different methods," Applied Energy, vol. 93, pp. 327-336, 2012.

[33] J. Kim, H. Ha, and S. Woo, "Dynamics of sediment disturbance by periodic artificial discharges from the world's largest tidal power plant," Estuarine, Coastal and Shelf Science, vol. 190, pp. 69 -79, 2017.

[34] P. Jeffcoate, D. Apsley, and P. Stansby, "Flow due to multiple jets downstream of a barrage: experiments, $3 \mathrm{~d}$ computational fluid dynamics, and depth-averaged modeling," Journal of Hydraulic Engineering, 2013.

[35] P. Jeffcoate, P. Stansby, and D. Apsley, "Flow and bed-shear magnification downstream of a barrage with swirl generated in ducts by stators and rotors," Journal of Hydraulic Engineering, vol. 143, no. 2 pp. 1-8, 2017 